\title{
The Functional Neuroanatomy of Tourette's Syndrome: An FDG-PET Study. I. Regional Changes in Cerebral Glucose Metabolism Differentiating Patients and Controls
}

Allen R. Braun, M.D., Brigitte Stoetter, M.D., Christopher Randolph, Ph.D., John K. Hsiao, M.D., Kate Vladar, M.D., Jeffrey Gernert, M.Phil., Richard E. Carson, Ph.D., Peter Herscovitch, M.D., and Thomas N. Chase, M.D.

Regional metabolic rates for glucose estimated using $\left[{ }^{18} \mathrm{~F}\right]$ fluorodeoxyglucose positron-emission tomography were compared in 16 drug-free patients with Tourette's syndrome (TS) and 16 age-and sex-matched normal volunteers. Tourette's syndrome patients were characterized by decreased normalized metabolic rates in paralimbic and ventral prefrontal cortices, particularly in orbitofrontal, inferior insular, and parahippocampal regions. Similar decreases were observed in subcortical regions, including the ventral striatum (nucleus accumbens/ventromedial caudate) and in the midbrain.
These changes were more robust and occurred with greater frequency in the left hemisphere. They were associated with concomitant bilateral increases in metabolic activity the supplementary motor, lateral premotor, and Rolandic cortices. Effects of prior exposure to neuroactive drugs did not account for these findings. These results suggest that an altered relationship between limbic-related regions of the cortex and striatum and cortical regions involved in the initiation of movement may play a role in the pathogenesis of this illness.

[Neuropsychopharmacology 9:277-291, 1993]
KEY WORDS: Tourette's syndrome; Positron-emission tomography; ${ }^{18}[\mathrm{~F}]$-fluorodeoxyglucose; Movement disorders; Basal ganglia; Premotor; Motor; Limbic
Gilles de la Tourette's syndrome (TS) is a complex neurologic disorder characterized by multiple motor and vocal tics, associated behavioral disturbances, and a chronic, fluctuating course. Although it is now clear that the disorder is genetically transmitted and is therefore likely to be associated with a structural or chemical diathesis, the brain mechanisms involved in the generation of symptoms have never been identified.

Tourette's syndrome is unambiguously a disorder of motor function. The multiple tics that are the hallmark of the syndrome must certainly involve brain regions that participate in the organization, initiation, and execution of movement. Nevertheless, the abnormal movements observed in, and experienced by, TS patients differ from involuntary movements (choreoathetosis, myoclonus, and dystonia) typically encountered in other hyperkinetic movement disorders (Lang 1991; Bliss 1980). Both motor and vocal tics are commonly 
preceeded by an experience of motivational tension and their expression is often followed by a sense of satisfaction or relief. The urge to tic is consciously experienced by the patient, who can suppress symptoms for a variable period of time, but will inevitably yield to what he feels "driven" to do. Symptoms may be described as alien or due to the operation of a "second will." The TS patient O. (Meige and Feindel 1907), describes a prototypical tic: "I know I am its author, but that cannot prevent my becoming its victim."

For these reasons, it appears likely that the pathogenesis of TS is not confined exclusively to the motor domain. The generation of symptoms may additionally involve brain regions associated with motivation and may reflect an abnormality of brain systems that are involved in the coupling of motivation and action specifically those which effect neural transmission from the limbic to the motor systems. Similarly, because the disorder is frequently associated with complex behavioral disturbances including obsessions and compulsions (Towbin, 1988), attentional difficulties (Comings and Comings, 1988), irritability, impulsivity, and self-injurious behavior (Riddle et al. 1988), and due to the presence of coprolalia in approximately half of all patients (Shapiro et al. 1973), the neuroanatomic substrate underlying symptoms in TS probably involves limbic as well as motor mechanisms.

But what regions of the brain are involved? The response of both motor and vocal tics to typical neuroleptic agents (Seignot 1961; Shapiro et al. 1973) and the demonstration of altered levels of homovanillic acid (HVA) in the cerebrospinal fluid (CSF) of some patients (Cohen et al. 1979; Singer et al. 1982) have suggested a primary role for the dopamine system in TS, perhaps involving increased sensitivity of dopamine receptors or postsynaptic mechanisms to which these receptors are coupled. However, models derived from pharmacologic information in this fashion inevitably suffer from a lack of anatomic specificity. Electrophysiologic studies demonstrate only nonspecific and nonlocalizing abnormalities (Van de Wetering et al. 1985; Sweet et al. 1973); in spite of reports of histologic or biochemical alterations in postmortem studies of TS brains (Balthasar 1956; Haber et al. 1986; Singer et al. 1990), no unequivocal neuropathologic changes have yet been associated with the disorder. The pathogenesis of Tourette's syndrome remains nearly as obscure as when the illness was first described more than a century ago (Gilles de la Tourette, 1885).

Positron-emission tomography (PET), using $\left[{ }^{18} \mathrm{~F}\right]-$ fluoro-2-deoxy-D-glucose (FDG), is a well-established method of characterizing functional activity in the living human brain (Phelps et al. 1979), which may be of particular value in illnesses without clear pathophysiologic features such as TS. Regional glucose metabolism-coupled to energy-requiring processes driven by the synaptic activity of neurons-appears to be a reliable index of functional activity within the central nervous system (Sokoloff 1992). In this study, we compared regional patterns of glucose metabolism in 16 drug-free individuals with typical TS and 16 age- and sex-matched control subjects in an attempt to characterize the functional neuroanatomy of this illness.

\section{SUBJECTS AND METHODS}

\section{Subjects}

Sixteen patients with TS, 14 males and two females, aged $33 \pm 7$ years (mean $\pm S D$; range 23 to 49 years) consented to participate in this study after providing informed consent. Fourteen patients were right handed and two, one male and one female, were left handed. The diagnosis of TS was confirmed by history and physical examination that excluded concurrent neurological, medical or unrelated psychiatric illnesses, and conformed to DSM-III-R criteria.

All patients were drug free at the time of scanning. Four patients had been taking dopamine receptor antagonists (haloperidol in three cases, pimozide in one) which were withdrawn at least 2 weeks (range 2 to 8 weeks) prior to the PET studies. Neuroleptics had been administered alone in two cases and in combination with other neuroactive medications (clonidine or imipramine) in two others. Two additional patients had been receiving nonneuroleptic medications alone, which were similarly withdrawn (in one patient clonidine was discontinued 4 weeks prior to the studies, and in anotherpatient, verapamil was discontinued 2 weeks prior to the studies).

Nine additional patients had a history of exposure to neuroleptic (haloperidol or chlorpromazine) or nonneuroleptic drugs (clonodine, imipramine or tranylcypromine) but had not received treatment for at least 1 year prior to these studies. One patient had never been exposed to neuroactive medications.

Sixteen normal volunteers, 11 males and five females, aged $34 \pm 10$ years (mean $\pm S D$; range 20 to 50 years) gave informed consent prior to participation. Fifteen subjects were right handed and one, a male, was left handed. Normal volunteers were without signifcant abnormalities at the time of general physical and neurologic examination and histories were negative for neurologic, medical, or psychiatric illness. No control subject used prescription or nonprescription drugs within the 2 weeks prior to scanning.

\section{PET Scans}

Positron-emission tomography scans were performed on the NeuroPET (Brooks et al. 1980), a seven-slice positron tomograph with an in-plane resolution of $6 \mathrm{~mm}$ 
to $7 \mathrm{~mm}$ and an axial resolution of $11 \mathrm{~mm}$ to $12 \mathrm{~mm}$ FWHM. Subjects were seated in a modified dental chair in a room with low ambient light and noise and allowed to acclimate to these surroundings. Eyes were patched and ears occluded before positioning the head within the scanner gantry. The plane of scanning was parallel to the canthomeatal line.

Over a period of 1 minute, $5 \mathrm{mCi}$ of FDG was injected intravenously, and 10-minute scans were initiated 30 to 45 minutes afterinfusion. Between each scan, the position of the patient's head was shifted within the gantry to extend the anatomic range sampled. Up to five interleaved scans were performed in each session, resulting in up to 35 slices per study. Arterialized venous blood (Phelps et al. 1979) was sampled throughout the duration of the study, from the arm opposite the site of FDG infusion, to provide measurements of plasma FDG and glucose concentration over time. $\mathrm{Pa}$ tients were asked to remain still during the course of the procedure. Although they were not explicitly instructed to suppress symptoms, vocal or motor tics were rarely observed during the period of FDG uptake.

Local cerebral metabolic rates for glucose were calculated according to the method of Brooks (1982), utilizing an equation that is relatively insensitive to errors in rate constants and that incorporates a term for glucose 6-phosphate dephosphorylation. A calculated attenuation correction was performed using a visually fitted outline of the scalp routinely apparent in the images. Scanner and gamma well counter were crosscalibrated using a phantom containing a solution of $\left.{ }^{[18} \mathrm{F}\right]$ fluoride.

\section{Image Analysis}

The results of PET studies are not infrequently inconsistent or difficult to replicate. This can be due to small patient numbers, differences in PET instrumentation or differences in the methods of data analysis. To minimize the last source of error and to enhance the reliability of our results, two technically distinct analytic approaches were applied to the PET data by separate investigators working independently, and the results of these analyses were compared.

Eight image planes (Figs. 1 and 2) were selected for analysis on the basis of recognizable cerebral landmarks identified in standard anatomic atlases (Damasio and Damasio 1989; Matsui and Hirano 1978; Duvernoy 1991). Each investigator, blinded to diagnosis as well as to the other's results, selected planes of section and employed distinct technical strategies for regional segmentation and estimation of glucose metabolic rates.

Analysis 1. Irregular regions of interest (ROIs) were applied in the cortex to maximize the use of anatomic detail available there and to include the entire range of metabolic data. Cortical-CSF and cortical-white matter boundaries were defined using thresholding techniques that located these edges at pixels with values of approximately $50 \%$ of the local cortical maximum. The segmented cortical mantle was then divided using a radial template that was adjusted with reference to major cortical landmarks (Fig. 3a). Regional perimeters were reduced by one pixel to decrease in-plane partial volume averaging and overlap between contiguous regions. These irregular cortical ROIs typically enclosed an area of between 25 and 75 pixels (100 to $300 \mathrm{~mm}^{2}$ ).

Circular ROIs, ranging in area from 8 to 12 pixels (32 to $48 \mathrm{~mm}^{2}$ ) depending upon the size of the anatomic area sampled, were applied to subcortical structures (basal ganglia, brainstem, and thalamus) and cortical regions (e.g., insula, medial temporal, and calcarine cortices) in which anatomic detail was less precise. Data reduction was performed in these instances by averaging values for structures within an image plane in which more than one small circular ROI was applied. This process yielded 164 ROIs in all-82 in each hemispherean average of approximately 20 per slice. In addition, brain size for each subject was estimated by averaging the cross-sectional areas of PET slices selected for use in analysis 1 .

Analysis 2. Regional metabolic rates were obtained by placing small circular ROIs on each image plane. These regions were of a uniform size (12 pixels, $48 \mathrm{~mm}^{2}$ ) and were clustered into a series of templates that were interactively adjusted to fit each subject's scan (Fig. 3b). Following application and coarse adjustment, the ROIs were independently translated and placed over the local maximum (activity peak) to decrease the effects of partial volume averaging (Hoffman et al. 1979). Successive ROIs were positioned no closer than within two pixels $(4 \mathrm{~mm})$ of the neighboring region, and local activity was similarly maximized. Two hundred forty six such ROls were placed on the eight planes of section. Data reduction was performed by averaging values to yield estimated metabolic rates for somewhat larger, yet anatomically discrete regions. This process reduced the number of regions to 112: 56 in each hemisphere, an average of 14 per slice.

Although the total number of regions in analyses 1 and 2 differed, templates were designed such that regions corresponded to coded areas in a common atlas (Damasio and Damasio (1989), which assigns both functional and cytoarchitectonic descriptors to anatomic areas of interest) for purposes of comparison.

\section{Statistical Analysis}

Both normalized and absolute metabolic rates were analyzed by univariate repeated-measures analyses of variance (ANOVA) - with diagnosis as the grouping 


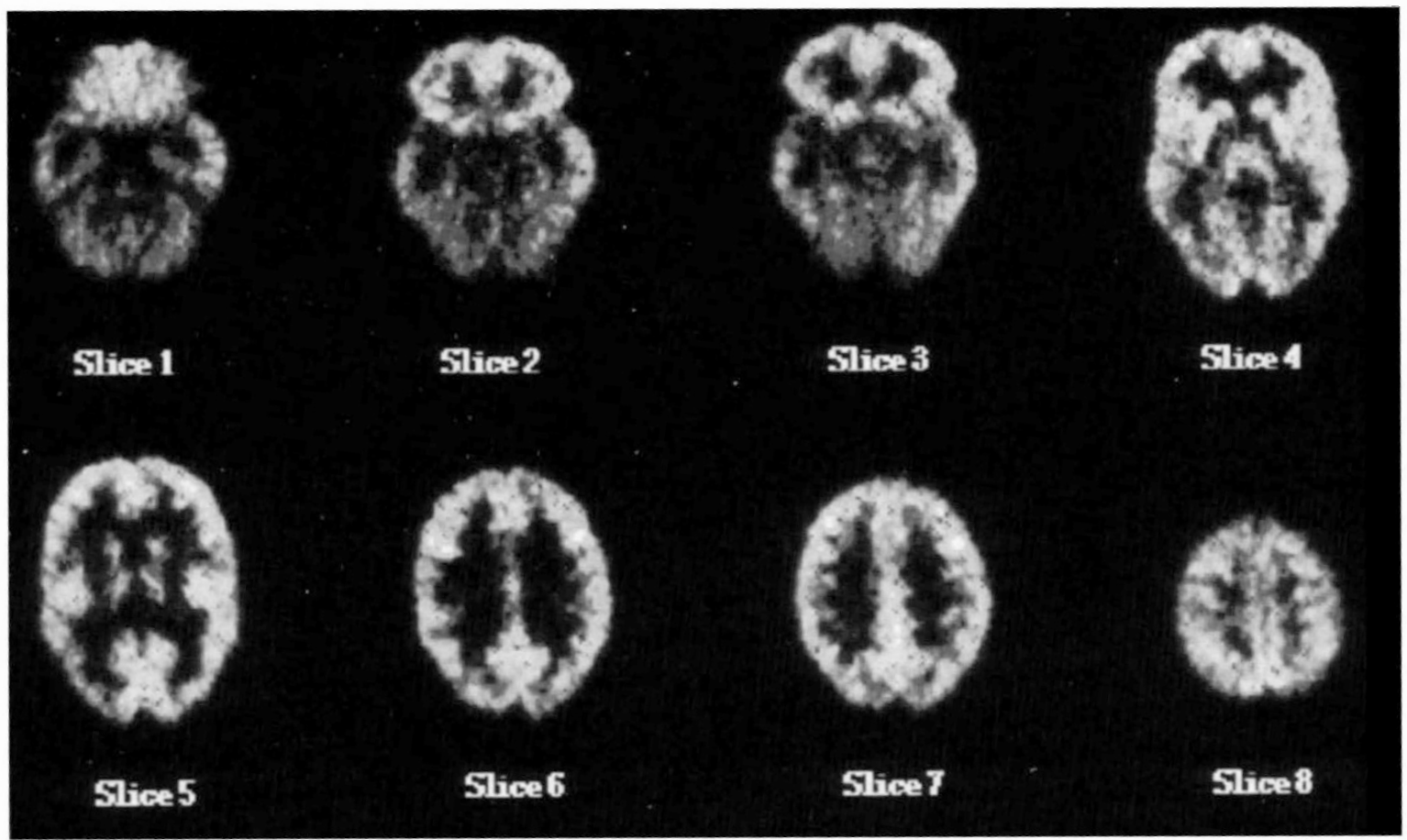

Figure 1. A typical PET data set, illustrating the eight planes of section chosen for analysis. Inferior regions (cerebellum, temporal pole, and gyrus rectus) are seen at the upper left, and superior regions (frontal and parietal convexities) are seen at lower right.

(between) factor and ROIs as the repeated (within) measures. Regional metabolic rates were normalized using whole-brain metabolic averages. In analysis 1 , whole-brain metabolic activity was estimated by averaging regional grey matter metabolic rates, weighting these for the area enclosed within each ROI. In analysis 2 , whole-brain metabolic activity was calculated by averaging metabolic rates in the 246 original circular ROIs.

If significant group differences were detected by repeated-measures ANOVA, individual regional metabolic rates were compared. Univariate factorial ANOVAs were used to compare regional means. Only significant differences that were detected in the same brain regions
Figure 2. Approximate location of PET planes of section (see Figs. 1 and 4) illustrated on a lateral representation of the brain in which cytoarchitectonic (Brodman) regions are indicated.

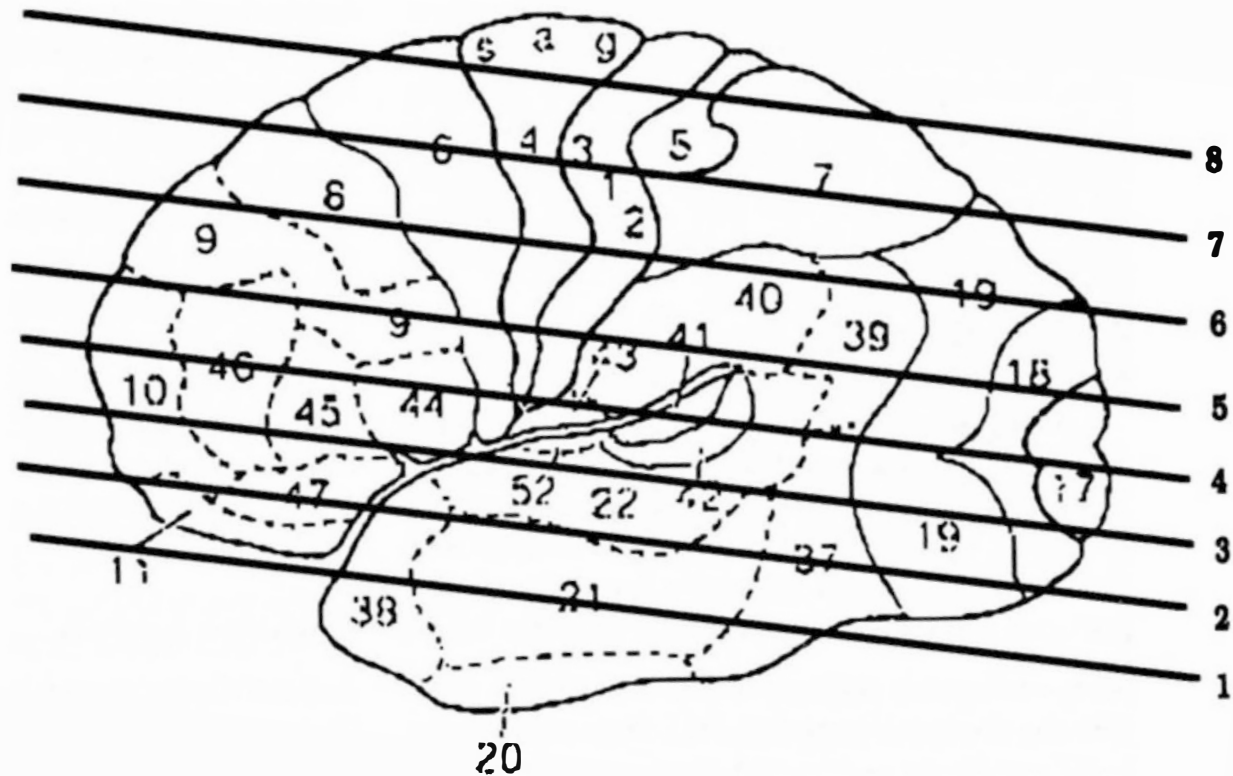



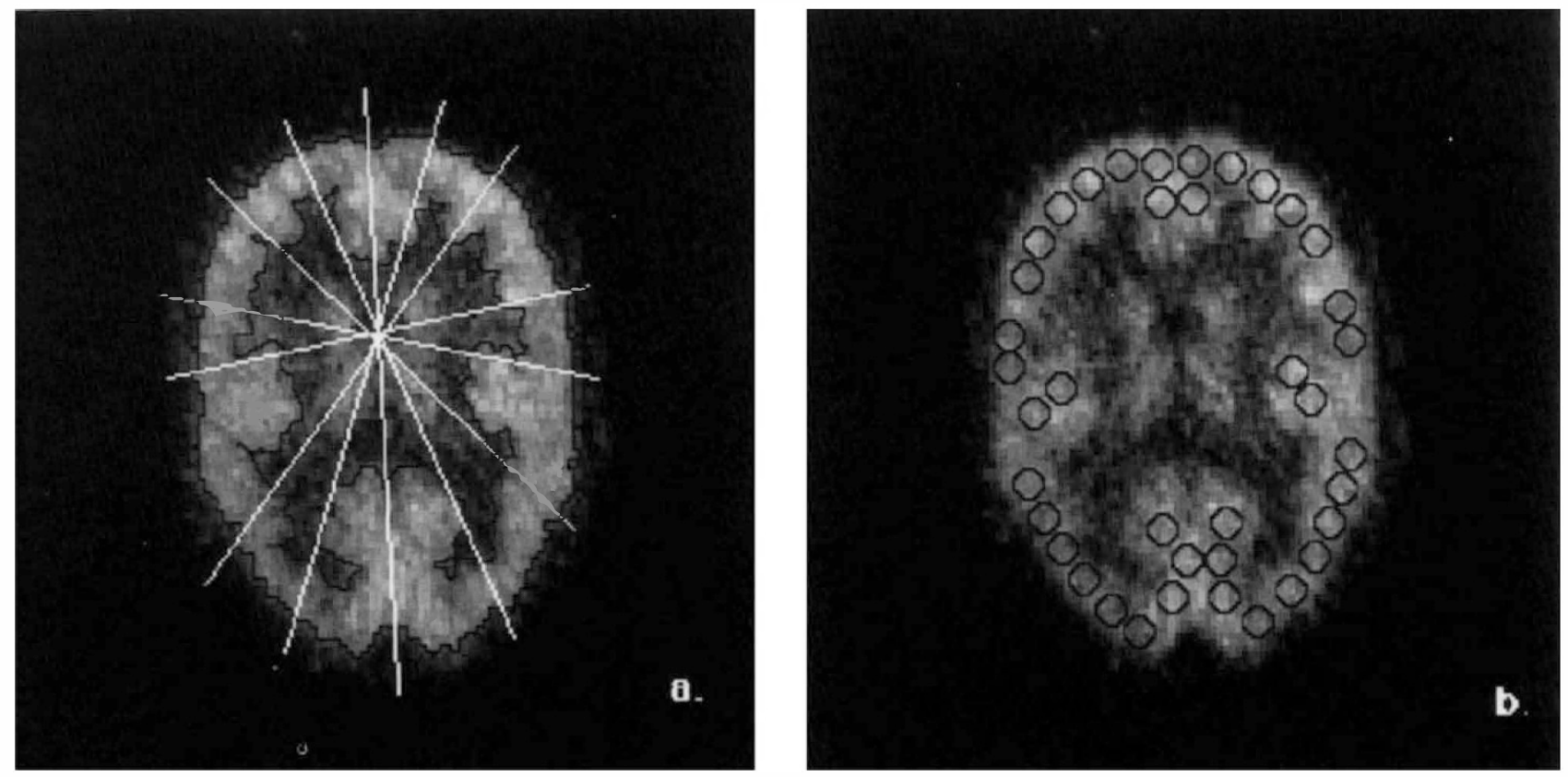

Figure 3. An illustration of the two methods by which ROIs were identified in this study. Analysis 1 . (a): Irregular ROIs are applied in the cortex to maximize the use of anatomic detail. Cortical-CSF and cortical-white matter boundaries are thresholded interactively; the cortical mantle is then divided using a radial template that is adjusted with reference to the midline and major cortical landmarks. Regional perimeters are reduced by one pixel to minimize in-plane partial volume averaging and overlap between contiguous regions. Analysis 2. (b): A template consisting of circular ROIs of $7.8 \mathrm{~mm}$ diameter is placed on the PET image; ROIs are then independently translated and placed over the local maximum (activity peak) to minimize the effects of partial volume averaging.

in both analyses were considered reliable indices of authentic group differences.

Analyses of variance were also used to examine the potential effect of prior drug exposure, brain size, and sex on estimated cerebral metabolic rates for glucose. In addition, regional metabolic rates were entered as variables in two-group discriminant analyses; for each data set, discriminant functions were derived through the linear combination of variables and used to reclassify the original set of cases with respect to diagnosis. Chi-squared tests were performed to evaluate the distribution of regional metabolic changes in right and left hemispheres.

\section{RESULTS}

\section{Comparison of Normalized Metabolic Rates in Patients and Controls}

Table 1 summarizes the results of overall repeatedmeasures ANOVAs performed on normalized metabolic rates in analyses 1 and 2 . In both cases, the diagnosis by region interaction term was significant $(p<.0001$, either instance) indicating that TS patients and control subjects can be distinguished by a pattern of differences in metabolic activity in specific regions of the brain.

These regions were identified by comparing metabolic rates in individual regions of interest. Figure 5 summarizes the group differences in normalized regional values derived in analysis 1 , and table 2 contains values derived in analysis 2 . In both cases, significant differences between patients and controls are indicated by asterisks.

Of the 32 significant differences detected in analysis 1 , and the 35 detected in analysis 2, 44 (22 pairs) were congruent: that is, differences, equal in sign, were identified within the same regions in both analyses. Differences that attained statistical significance in one analysis only were accompanied by matching trends $(p<.2)$ in the other analysis $70 \%$ of the time ( 16 of 23 remaining cases), and significant differences were commonly detected, in the other analysis, in contiguous, functionally related, or homologous contralateral ROIs. Nevertheless, as outlined above, only significant differences that were identified in the same regions in both analyses were considered reliable, that is, robust enough to potentially represent genuine differences between patients and controls. These regions are listed in Table 3. In this table, differences located in two anatomically contiguous, functionally related regions (e.g., superior lateral premotor cortex 1 and 2) are grouped together as are right and left hemispheral ROIs.

These reliable or congruent changes can be summarized as follows. Tourette's syndrome patients, in the present study, appear to be characterized by lower 


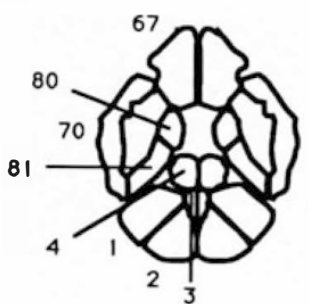

Slice 1

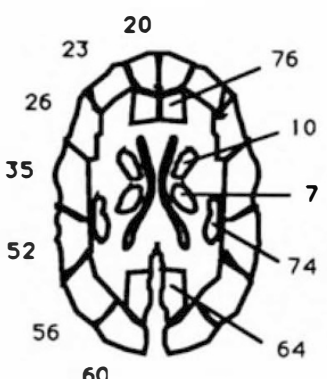

Slice 5

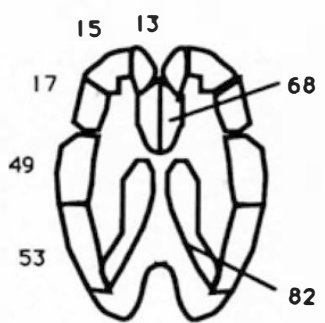

Slice 2

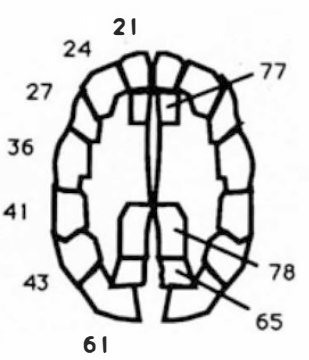

Slice 6

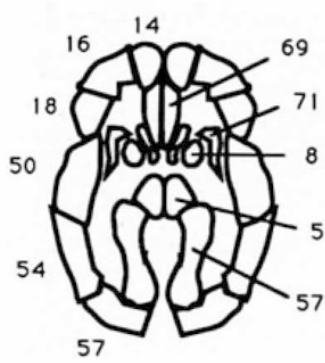

Slice 3

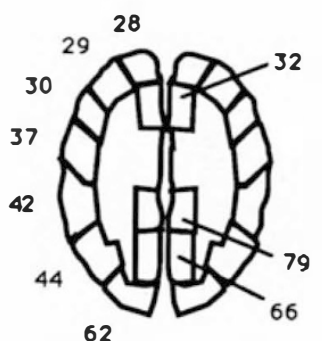

Slice 7

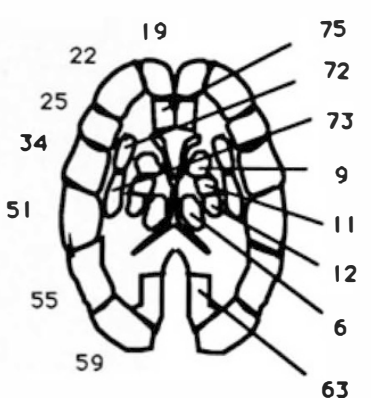

Slice 4

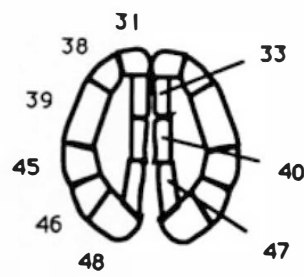

Slice 8

\section{Regions of Interest}

\begin{tabular}{|c|c|}
\hline $\begin{array}{l}1 \\
9\end{array}$ & 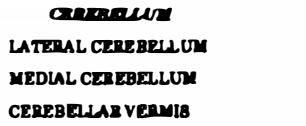 \\
\hline 4 & $\begin{array}{l}\text { eaversm } \\
\text { PONB } \\
\text { MIDBenN }\end{array}$ \\
\hline ? & $\begin{array}{l}\text { Tanchets } \\
\text { VENTHL TBULANUB } \\
\text { DORgAL TBALANUB }\end{array}$ \\
\hline $\begin{array}{l}8 \\
10 \\
11 \\
18\end{array}$ & 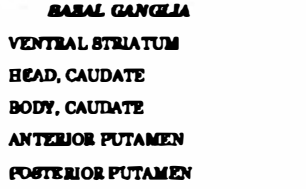 \\
\hline $\begin{array}{l}13 \\
14 \\
18 \\
16 \\
17 \\
18 \\
10 \\
20 \\
21 \\
2 \\
25 \\
21 \\
28 \\
28 \\
27\end{array}$ & 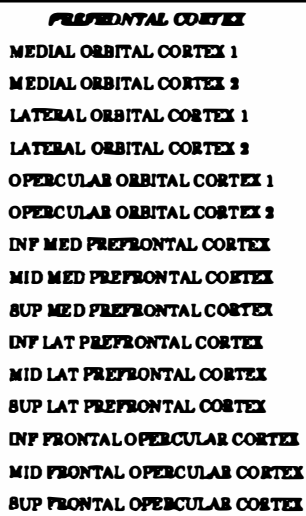 \\
\hline
\end{tabular}

\begin{tabular}{|c|c|}
\hline & RENTAL EOTOR COETET \\
\hline 2s & DNP LAT PREMOTOR CORTEY 1 \\
\hline 20 & DNF LAT PREMOTOR CORTEX 2 \\
\hline $\boldsymbol{s}$ & DNF LAT PREMOTOR CORTEX ? \\
\hline 91 & SUP LAT PRDAOTOR CORTEX \\
\hline 32 & ANTEROR BMA \\
\hline ss & POETEvor am \\
\hline s4 & DNF ROLANDIC CORTEX 1 \\
\hline 36 & DNF ROLUNDIC CORTEX 2 \\
\hline o & DNF ROLANDIC CORTEY J \\
\hline 37 & BUP ROLANDIC CORTEX 1 \\
\hline ss & BUP ROLANDIC CORTEX 2 \\
\hline $\boldsymbol{x}$ & BUP ROLANDIC CORTEX 3 \\
\hline 40 & PARACENTRAL LOBULE \\
\hline & PAPITAR COATS \\
\hline 41 & GOREMPGDNAL OYRUB 1 \\
\hline 42 & BUPRUMUEDNAL GTRUB 2 \\
\hline$\infty$ & ANGULAR GYRUB 1 \\
\hline 4 & ANGULAR GYRUS \\
\hline 48 & ANT LAT SUPENOR PANETAL LOBULE \\
\hline 4 & POET LAT BUPEEOR PADETAL LOBULE \\
\hline 47 & ANT MED BUREROR PANETAL LOBCLE \\
\hline 4 & POET MED BUPREIOR PARIETAL LOBULE \\
\hline & neroze cons \\
\hline 40 & ANTEROR BUPRPIOR TEMPORAL CORTEX I \\
\hline$\infty$ & ANTEDOR BUPESIOR TEMPORAL CORTEX 2 \\
\hline $\mathbf{B 1}$ & POATXROR BUPEDOR TEMPORAL CORTEX I \\
\hline 8 & POATXPOR BUPEDOR TEMPORAL CORTEX 2 \\
\hline $\mathbf{O}$ & ANTEDOR DNEROR TEMPORL CORTE 1 \\
\hline $\boldsymbol{u}$ & ANTEWOR DNEENOR TEMPORAL COTTET I \\
\hline 8 & ANTEDOR DNEROR TEMPORAL COETX 1 \\
\hline 6 & POATEROR NFARIOR TEMPORLL CORTEX 2 \\
\hline
\end{tabular}

\begin{tabular}{|c|c|}
\hline & OCCIPITA coomer \\
\hline 67 & FUBIFORY (OOCIPTTOTEMPORNW GYRUB \\
\hline os & DNF LAT OOCIPITAL CORTEX 1 \\
\hline so & DNF LAT OOCIPITAL CORTEX 2 \\
\hline$\infty$ & MID LAT OCCIPITAL CORTEX \\
\hline 01 & BUP LAT OOCIPTTAL CORTEX 1 \\
\hline$\infty$ & BUP LAT OOCIPTTAL CORTEX 2 \\
\hline os & DNF CALCNINE CORTEX 1 \\
\hline ด & DNF CALCARDE COR TEX 2 \\
\hline es & BUP CALCARNE CORTEX I \\
\hline $\boldsymbol{6}$ & BUP CALCARENE CORTEX 2 \\
\hline
\end{tabular}

\begin{tabular}{|c|c|}
\hline & PATPOTERCLL HMAC \\
\hline 07 & GYRUB RECTUP \\
\hline es & CAUDAL ORBITAL CORTEX 1 \\
\hline$\infty$ & CAUOAL ORBITAL CORTEX 2 \\
\hline 70 & TEMPORAL POLAR CORTEX \\
\hline 71 & DNFEYOR DNBULA \\
\hline 72 & ANTEROOR INBULA \\
\hline 79 & FOCTENOR DNBULA \\
\hline 74 & BUPETUOR DNBULA \\
\hline
\end{tabular}

\begin{tabular}{|c|c|}
\hline & Asciacodich HATC \\
\hline 78 & DNF ANTENOOR CDNGULATE \\
\hline 78 & MID ANTEPYOR CINGULATE \\
\hline $\boldsymbol{\pi}$ & SUPANTERIOR CDNGULATE \\
\hline 78 & DNF FOETSPIOR CDNGULATE \\
\hline ד & EUP POSTETYOR CDNGULATE \\
\hline$\infty$ & NYYGDALA-BIPPOCNMPUB \\
\hline $\mathbf{8 1}$ & PARARIPPOC. OYRUS - HIPPOCAMPUB 1 \\
\hline 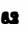 & PARABIPPOC GYRUB - EUPPOCAMPUB I \\
\hline
\end{tabular}

Figure 4. A representation of the ROIs derived in analysis 1 for the eight PET planes of section (Figs. 1 and 2). Numbers refer to the categorical list of region names contained in the legend. 
Table 1. ANOVA for Regional Metabolic Rates in TS Patients and Normal Volunteers

\begin{tabular}{lrcrc}
\hline Source & $d f$ & SS & F-test & $p$-value \\
\hline Analysis 1 & & & & \\
$\quad$ Diagnosis & 1 & 0.014 & 0.79 & NS \\
Region & 163 & 63.03 & 60.26 & $p<.0001$ \\
$\quad$ Region x Diagnosis & 163 & 1.544 & 1.48 & $p<.0001$ \\
Analysis 2 & & & & \\
$\quad$ Diagnosis & 1 & 1.505 & 2.51 & NS \\
Region & 111 & 72.979 & 81.31 & $p<.0001$ \\
Region $\times$ Diagnosis & 111 & 3.019 & 3.36 & $p<.0001$ \\
\hline
\end{tabular}

Diagnosis $=$ Grouping Factor; Normalized Regional Metabolic Rates $=$ Repeated Measures, SS $=$ sums of squares

relative metabolic rates in inferior, limbic-associated areas of the brain - in paralimbic and ventral prefrontal regions, in the striatum and the brainstem - and by higher relative metabolic rates in superior, sensorimotor cortices.

In the inferior prefrontal and paralimbic regions of the cortex, decreased metabolic rates are observed in orbitofrontal, but not dorsolateral prefrontal cortices; patients are characterized by reductions in metabolic activity in the inferior paralimbic regions of the insula and in the related caudal orbital, but not cingulate cortices. Decreases in metabolic activity are observed in the parahippocampal (entorhinal) region as well. In the basal ganglia, decreased metabolic activity in TS patients is most pronounced in the limbic-associated region of the ventral striatum. This region of interest, at the $\mathrm{z}$-axis resolution of our scanner, will include metabolic information from the ventral striatum proper (nucleus accumbens) and ventromedial regions of the caudate nucleus. Metabolic activity is decreased in the left anterior putamen in patients as well, but this difference is not as robust as that observed in the ventral striatum. Significant decreases in metabolic activity are also observed in the midbrain. In the superior sensorimotor cortices, significant increases in metabolic rates for glucose in TS patients are clustered in the frontal motor cortices-including supplementary motor, lateral premotor, and Rolandic regions.

There appears, in addition, to be a hemispheral asymmetry in the distribution of decreased metabolic rates: significant decreases are more frequent, or more robust, in prefrontal, limbic, and striatal regions of the left hemisphere $\left(\chi^{2}=10.32, p<.005\right.$, analysis $1 ; \chi^{2}=$ $9.33, p<.005$, analysis 2$)$. The increases in normalized cerebral metabolic rates for glucose (CMRglu) in dorsal sensorimotor ROIs, on the other hand, are not signifcantly lateralized.

All of these relative differences are depicted in Figure 5. Metabolic activity in inferior prefrontal cortices, striatum and brainstem of an individual TS patient and control are compared in Figure 6.

\section{Discriminant Analyses}

To determine whether or not cerebral metabolism in this circumscribed set of regions is sufficient to accurately predict diagnosis, two-group discriminant analyses were performed. A separate analysis was conducted for each data set, but only regional values that attained statistical significance in both analyses were entered as discriminating variables.

Discriminant functions derived using this set of regional metabolic rates were used to reclassify the original set of cases to determine how often the correct diagnosis would be assigned. In both cases, metabolic rates in the limited, "reliable" set of regions assigned each subject to the correct diagnostic category without error. This indicates that metabolic rates in only those subcortical, ventral limbic, premotor, and Rolandic regions listed in Table 3 are needed to characterize TS patients and reliably distinguish them from normal controls.

\section{Comparison of Absolute Metabolic Rates in Patients and Controls}

When absolute metabolic rates for glucose are compared, analyses 1 and 2 appear to be sensitive to changes occurring at opposite ends of the metabolic spectrum. In either analysis, when mean global grey matter metabolic rates were compared, no significant differences between patients and normal volunteers were observed (analysis 1: TS $=8.46 \pm 1.29 \mathrm{mg}$ glucose $/ 100 \mathrm{~g} / \mathrm{min}$, normals $=8.69 \pm 1.45$; analysis 2 : $\mathrm{TS}=8.72 \pm 1.28$ $\mathrm{mg}$ glucose $/ 100 \mathrm{~g} / \mathrm{min}$, normals $=8.54 \pm 1.32$ ) .

Nevertheless, repeated-measures ANOVAs utilizing absolute regional metabolic rates generated a significant diagnosis by region interaction term in both instances (analysis 1: $F=1.30 ; d f=1,163 ; p<.007$; analysis $2: F=1.96 ; d f=1,111 ; p<.0001)$, indicating that although global grey matter metabolic rates do not differ, TS patients and controls can again be distinguished by a pattern of differences in metabolic activity in specific regions of the brain. These brain regions were identified by comparing individual regional CMRglu values by univariate ANOVA. Regions in which differences ( $p<.05$, uncorrected) were observed appeared to cluster in different areas of the brain, depending on the analytic method used.

Analysis 1 appeared to be more sensitive in the detection of decreased absolute regional metabolic rates in TS patients. Decreases in absolute CMRglu were observed in inferior limbic cortical and subcortical ROIs (left and right medial orbital, right caudal orbital, and left lateral orbitofrontal cortices; left inferior insular cortex; and left and right parahippocampal regions; and left and right midbrain).

Analysis 2, on the other hand, appeared to be more sensitive in the detection of increased absolute 
Table 2. Normalized Regional Metabolic Rates: Tourettes vs. Normal Volunteers (Analysis 2)

\begin{tabular}{|c|c|c|c|c|}
\hline \multirow[b]{2}{*}{ Regions of Interest } & \multicolumn{2}{|c|}{ Normal Volunteers } & \multicolumn{2}{|c|}{ Tourette's Syndrome } \\
\hline & Left & Right & Left & Right \\
\hline \multicolumn{5}{|l|}{ Subcortical regions } \\
\hline Cerebellar hemisphere & $0.862 \pm 0.025$ & $0.846 \pm 0.021$ & $0.827 \pm 0.014$ & $0.844 \pm 0.017$ \\
\hline Dentate nucleus & $0.718 \pm 0.022$ & $0.738 \pm 0.026$ & $0.752 \pm 0.025$ & $0.743 \pm 0.026$ \\
\hline Pons & $0.645 \pm 0.019$ & $0.641 \pm 0.021$ & $0.597 \pm 0.013^{\star}$ & $0.601 \pm 0.013$ \\
\hline Midbrain & $0.848 \pm 0.021$ & $0.842 \pm 0.020$ & $0.759 \pm 0.016^{\star \star \star}$ & $0.757 \pm 0.018^{\star \star \star}$ \\
\hline Ventral striatum & $1.055 \pm 0.025$ & $1.019 \pm 0.024$ & $0.947 \pm 0.014^{\star \star \star \star}$ & $0.950 \pm 0.015^{\star}$ \\
\hline Caudate, head & $1.183 \pm 0.024$ & $1.173 \pm 0.025$ & $1.104 \pm 0.018$ & $1.070 \pm 0.024$ \\
\hline Caudate, body & $1.042 \pm 0.036$ & $1.039 \pm 0.039$ & $0.968 \pm 0.021$ & $0.966 \pm 0.033$ \\
\hline Anterior putamen & $1.261 \pm 0.028$ & $1.239 \pm 0.022$ & $1.121 \pm 0.027^{\star}$ & $1.133 \pm 0.019^{\star}$ \\
\hline Posterior putamen & $1.125 \pm 0.021$ & $1.137 \pm 0.033$ & $1.039 \pm 0.018$ & $1.049 \pm 0.017$ \\
\hline Thalamus & $1.104 \pm 0.033$ & $1.144 \pm 0.031$ & $1.087 \pm 0.020$ & $1.091 \pm 0.023$ \\
\hline White matter & $0.297 \pm 0.013$ & $0.293 \pm 0.012$ & $0.287 \pm 0.007$ & $0.296 \pm 0.012$ \\
\hline \multicolumn{5}{|l|}{ Prefrontal cortices } \\
\hline Medial orbital cortex & $0.994 \pm 0.039$ & $0.928 \pm 0.047$ & $0.905 \pm 0.019^{\star}$ & $0.889 \pm 0.030$ \\
\hline Lateral orbital cortex & $1.102 \pm 0.041$ & $1.107 \pm 0.030$ & $0.975 \pm 0.023^{\star *}$ & $1.020 \pm 0.027^{\star}$ \\
\hline Opercular orbital cortex & $1.058 \pm 0.033$ & $1.041 \pm 0.026$ & $0.910 \pm 0.018^{\star \star \star \star \star}$ & $0.978 \pm 0.027$ \\
\hline Inf medial prefrontal cortex & $1.110 \pm 0.035$ & $1.149 \pm 0.021$ & $1.115 \pm 0.033$ & $1.143 \pm 0.033$ \\
\hline Inf dorsolat prefrontal cortex & $1.126 \pm 0.029$ & $1.103 \pm 0.021$ & $1.059 \pm 0.021$ & $1.103 \pm 0.019$ \\
\hline Sup medial prefrontal cortex & $1.129 \pm 0.023$ & $1.163 \pm 0.019$ & $1.148 \pm 0.017$ & $1.180 \pm 0.021$ \\
\hline Sup dorsolat prefrontal cortex & $1.139 \pm 0.023$ & $1.165 \pm 0.017$ & $1.121 \pm 0.021$ & $1.158 \pm 0.020$ \\
\hline \multicolumn{5}{|l|}{ Frontal motor cortices } \\
\hline Anterior SMA & $1.070 \pm 0.022$ & $1.019 \pm 0.023$ & $1.112 \pm 0.018^{\star}$ & $1.086 \pm 0.019^{\star}$ \\
\hline Inf lateral premotor cortex 1 & $1.040 \pm 0.020$ & $1.049 \pm 0.018$ & $1.121 \pm 0.013^{\star \star \star \star}$ & $1.144 \pm 0.016^{\star \star \star \star \star}$ \\
\hline Inf lateral premotor cortex 2 & $1.131 \pm 0.018$ & $1.129 \pm 0.017$ & $1.181 \pm 0.016^{\star}$ & $1.201 \pm 0.017^{\star \star}$ \\
\hline Inf Rolandic cortex & $0.951 \pm 0.024$ & $0.944 \pm 0.020$ & $0.976 \pm 0.027$ & $1.002 \pm 0.020^{\star}$ \\
\hline Posterior SMA 1 & $1.003 \pm 0.027$ & $0.995 \pm 0.027$ & $1.122 \pm 0.026^{\star \star \star}$ & $1.014 \pm 0.029^{\star \star}$ \\
\hline Posterior SMA 2 & $1.046 \pm 0.027$ & $1.012 \pm 0.021$ & $1.127 \pm 0.024^{\star}$ & $1.073 \pm 0.023$ \\
\hline Sup lateral premotor cortex 1 & $1.075 \pm 0.024$ & $1.060 \pm 0.024$ & $1.133 \pm 0.017^{\star}$ & $1.138 \pm 0.020^{\star}$ \\
\hline Sup lateral premotor cortex 2 & $1.114 \pm 0.019$ & $1.085 \pm 0.029$ & $1.188 \pm 0.025^{\star}$ & $1.182 \pm 0.019^{\star \star}$ \\
\hline Sup rolandic cortex & $1.020 \pm 0.020$ & $0.955 \pm 0.028$ & $1.098 \pm 0.021^{\star \star}$ & $1.080 \pm 0.022^{\star \star \star}$ \\
\hline Ant paracentral lobule & $0.985 \pm 0.017$ & $0.972 \pm 0.021$ & $1.019 \pm 0.033$ & $0.962 \pm 0.035$ \\
\hline Post paracentral lobule & $0.950 \pm 0.022$ & $0.939 \pm 0.029$ & $0.980 \pm 0.035$ & $0.911 \pm 0.037$ \\
\hline \multicolumn{5}{|l|}{ Parietal cortices } \\
\hline Ant inferior parietal lobule & $0.999 \pm 0.012$ & $1.008 \pm 0.017$ & $0.985 \pm 0.018$ & $1.030 \pm 0.017$ \\
\hline Post inferior parietal lobule & $0.943 \pm 0.018$ & $0.924 \pm 0.025$ & $0.930 \pm 0.012$ & $0.921 \pm 0.021$ \\
\hline Ant lat superior parietal lobule & $1.007 \pm 0.016$ & $0.987 \pm 0.025$ & $1.033 \pm 0.017$ & $1.025 \pm 0.019$ \\
\hline Post lat superior parietal lobule & $0.922 \pm 0.024$ & $0.918 \pm 0.018$ & $0.945 \pm 0.021$ & $0.941 \pm 0.025$ \\
\hline Ant med sup parietal lobule & $1.093 \pm 0.031$ & $1.076 \pm 0.027$ & $1.145 \pm 0.022$ & $1.092 \pm 0.020$ \\
\hline Post med sup parietal lobule & $1.148 \pm 0.025$ & $1.087 \pm 0.031$ & $1.165 \pm 0.033$ & $1.171 \pm 0.033$ \\
\hline \multicolumn{5}{|l|}{ Temporal cortices } \\
\hline Ant superior temporal & $1.017 \pm 0.020$ & $1.029 \pm 0.018$ & $0.948 \pm 0.012^{\star \star}$ & $1.001 \pm 0.014$ \\
\hline Ant inferior temporal & $0.967 \pm 0.015$ & $0.986 \pm 0.019$ & $0.940 \pm 0.020$ & $0.959 \pm 0.014$ \\
\hline Post superioral temporal & $1.040 \pm 0.030$ & $1.059 \pm 0.024$ & $1.010 \pm 0.013$ & $1.071 \pm 0.016$ \\
\hline Post inferior temporal & $1.031 \pm 0.020$ & $1.056 \pm 0.016$ & $0.977 \pm 0.013^{\star}$ & $1.026 \pm 0.019$ \\
\hline \multicolumn{5}{|l|}{ Occipital cortices } \\
\hline Inf lateral occipital & $0.848 \pm 0.020$ & $0.850 \pm 0.023$ & $0.801 \pm 0.012^{\star}$ & $0.813 \pm 0.018$ \\
\hline Mid lateral occipital & $0.831 \pm 0.028$ & $0.826 \pm 0.020$ & $0.814 \pm 0.017$ & $1.026 \pm 0.014$ \\
\hline Sup lateral occipital & $0.872 \pm 0.020$ & $0.848 \pm 0.020$ & $0.864 \pm 0.018$ & $0.837 \pm 0.015$ \\
\hline Inf calcarine cortex & $0.915 \pm 0.030$ & $0.908 \pm 0.028$ & $0.863 \pm 0.027$ & $0.878 \pm 0.025$ \\
\hline Mid calcarine cortex & $1.041 \pm 0.027$ & $0.980 \pm 0.026$ & $1.004 \pm 0.019$ & $0.970 \pm 0.016$ \\
\hline Sup calcarine cortex & $1.192 \pm 0.025$ & $1.092 \pm 0.026$ & $1.161 \pm 0.025$ & $1.130 \pm 0.024$ \\
\hline \multicolumn{5}{|l|}{ Limbic regions } \\
\hline Anterior insula & $1.139 \pm 0.032$ & $1.156 \pm 0.035$ & $1.044 \pm 0.018$ & $1.019 \pm 0.021$ \\
\hline Posterior insula & $0.999 \pm 0.014$ & $1.030 \pm 0.027$ & $0.899 \pm 0.012^{\star \star}$ & $0.889 \pm 0.013$ \\
\hline Inferior insula & $1.172 \pm 0.028$ & $1.149 \pm 0.029$ & $1.029 \pm 0.019^{\star \star \star \star \star}$ & $1.083 \pm 0.029$ \\
\hline Inf temp cortex/temporal pole & $0.947 \pm 0.015$ & $0.978 \pm 0.022$ & $0.875 \pm 0.021^{\star \star}$ & $0.957 \pm 0.018$ \\
\hline Gyrus rectus & $1.029 \pm 0.037$ & $0.993 \pm 0.027$ & $0.980 \pm 0.025$ & $0.957 \pm 0.032$ \\
\hline Caudal orbital cortex & $1.048 \pm 0.036$ & $1.022 \pm 0.030$ & $0.978 \pm 0.015$ & $0.955 \pm 0.019$ \\
\hline Parahippocampal region & $0.907 \pm 0.013$ & $0.920 \pm 0.014$ & $0.829 \pm 0.011^{\star \star \star \star \star \star}$ & $0.841 \pm 0.016^{\star \star \star}$ \\
\hline Inf anterior cingulate & $1.114 \pm 0.026$ & $1.047 \pm 0.029$ & $1.083 \pm 0.019$ & $1.060 \pm 0.020$ \\
\hline Sup anterior cingulate & $1.123 \pm 0.021$ & $1.060 \pm 0.017$ & $1.108 \pm 0.012$ & $1.069 \pm 0.017$ \\
\hline Inf posterior cingulate & $1.093 \pm 0.038$ & $1.073 \pm 0.025$ & $1.024 \pm 0.028$ & $1.024 \pm 0.023$ \\
\hline Sup posterior cingulate & $1.158 \pm 0.029$ & $1.122 \pm 0.030$ & $1.144 \pm 0.032$ & $1.095 \pm 0.027$ \\
\hline
\end{tabular}

${ }^{\star} p<.05,{ }^{\star \star} p<.01,^{\star \star \star} p<.005,{ }^{\star \star \star \star} p<.001,{ }^{\star \star \star \star \star} p<.0005$ versus normal volunteers.

Data are presented as mean \pm standard error. 


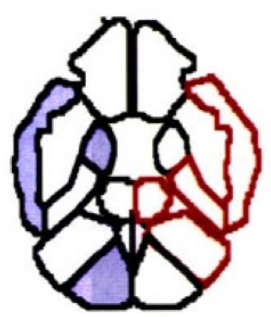

Slice 1

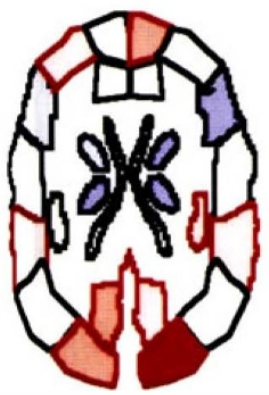

Slic 5

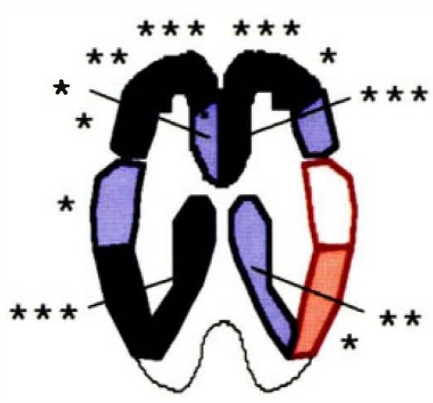

Slice 2

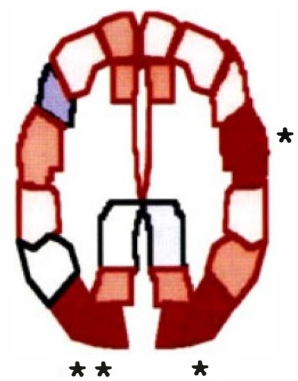

Slice 6

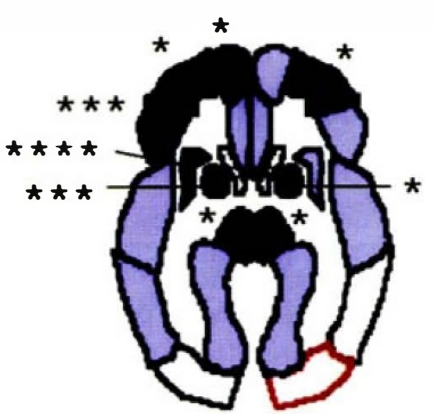

Slice 3

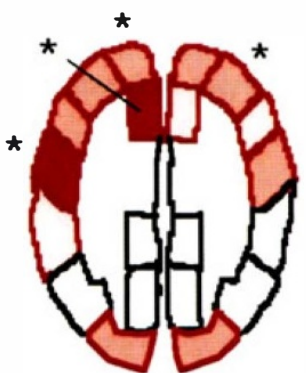

Slice 7

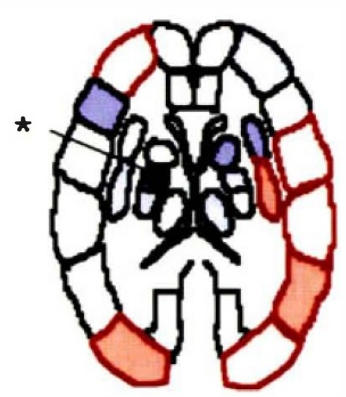

Slice 4

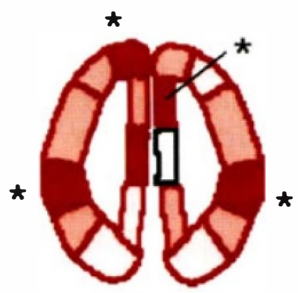

Slice 8

Figure 5. A representation of regional differences between normalized metabolic rates computed for TS patients and normal volunteers in analysis 1 . Blue indicates a decrease in regional metabolism in TS patients, and red indicates an increase. The magnitude of the differences between group means is indicated by the degree of stippling: changes of $2 \%$ to $4 \%$ are indicated by light stippling; $4 \%$ to $7 \%$ by moderate stippling; $7 \%$ to $10 \%$ by dense stippling; and greater than $10 \%$ by solid color. Significant differences are indicated by asterisks: ${ }^{\star} p<.05,{ }^{\star \star} p<.01,{ }^{\star \star \star} p<.005$, and ${ }^{\star \star \star \star} p<.0001$. Regions are identified by referring to Figure 4.

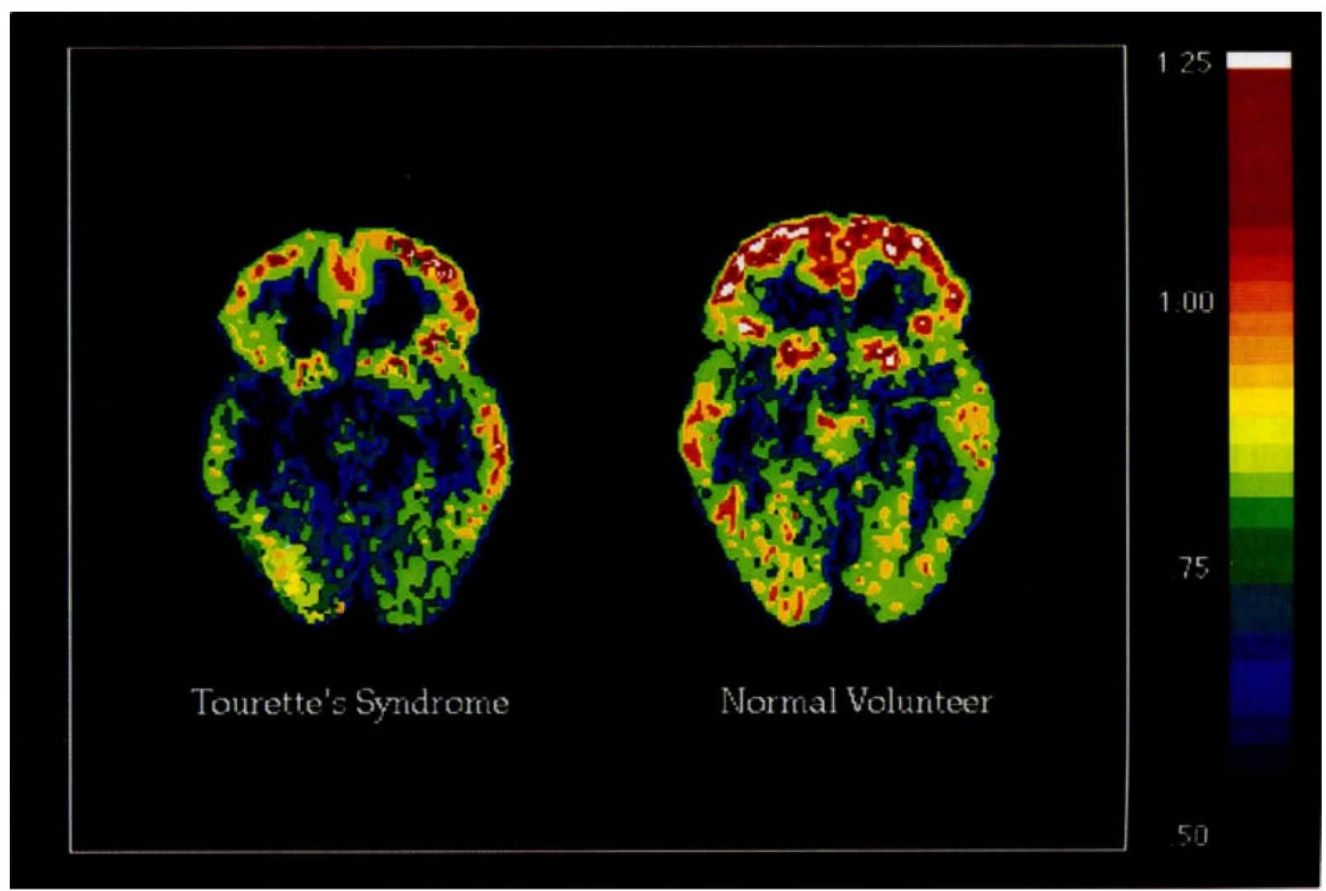

Figure 6. Positron-emission tomography data, corresponding to image plane 3 (see figs. 1, 2, 4, and 5), from a TS patient (left) and a normal volunteer (right). Images represent normalized metabolic rates and were produced by dividing each pixel by the subject's global, whole-brain gray matter metabolic rate, calculated as outlined in the Methods section. Relative decreases in metabolic activity, which were significant when the entire cohort of patients and controls were evaluated, can be seen in the ventral striatum, inferior insula and orbitofrontal cortices, particularly in the left hemisphere, and in the midbrain. 
Table 3. Regions in Which Normalized CMRglu Differentiates Patients and Controls in Both Analyses 1 and 2

\begin{tabular}{lc}
\hline Decreases & Increases \\
\hline $\begin{array}{l}\text { Brainstem } \\
\text { Midbrain }\end{array}$ & $\begin{array}{c}\text { Premotor cortices } \\
\text { Superior lateral premotor } \\
\text { cortex } \\
\text { Inferior lateral premotor } \\
\text { cortex } \\
\text { Ventral striatum } \\
\text { Putamen }\end{array}$ \\
$\begin{array}{ll}\text { Anterior SMA } \\
\text { Posterior SMA }\end{array}$ \\
$\begin{array}{l}\text { Prefrontal/Paralimbic cortices } \\
\text { Caudal orbital cortex }\end{array}$ & $\begin{array}{c}\text { Primary sensorimotor } \\
\text { Medial orbital cortex }\end{array}$ \\
Lateral orbital cortex & Inferior Rolandic cortex \\
Opercular orbital cortex & \\
Inferior insula & \\
Parahippocampal region & \\
\hline
\end{tabular}

regional metabolic rates in TS patients. Increased absolute CMRglu values were found in the superior frontal cortices, in premotor and Rolandic ROIs (inferior lateral premotor cortices in both right and left hemispheres and posterior supplementary motor and superior Rolandic cortices on the right). In a single instance, a significant decrease in CMRglu was detected in TS patients in the left lateral orbital cortex.

Coefficients of variation for absolute regional metabolic rates did not differ significantly between analyses 1 and 2 . In every case, significant differences detected in one analysis were accompanied by matching trends in the other.

The differing sensitivities of analyses 1 and 2 may be related to methodologic variations and perhaps reflect differences in the effective sample space applied in each analysis. For example, whereas the technique employed in analysis 1 utilizes all of the data in the PET images (see Fig. 3), in-plane partial volume averaging will have a larger effect on estimated metabolic rates, that is, will result in more statistical bias. On the other hand, although analysis 2 utilizes a method that is designed to minimize in-plane partial volume averaging (Hoffman et al. 1979) and produces results that are more stable, it samples only a relatively small extent of the anatomic area in question. In any case, as outlined above, when metabolic rates are normalized, controlling for intersubject differences in CMRglu and thereby reducing group variances, the results of analyses 1 and 2 converge.

\section{Effects of Drug Treatment in TS Patients}

The potential effect of recent exposure to neuroactive medications in TS patients was evaluated. Patients were grouped according to drug history: subjects who had undergone acute withdrawal from medications ( 2 to 8 weeks prior to study; $n=6$ ) were compared with those that had been drug-free for a year or more or who had never received neuroactive medications $(n=10)$. The effect of acute withdrawal from dopamine receptor antagonists $(n=4)$ was tested independently.

When withdrawal from medications per se was examined, an effect on whole-brain metabolic rates for glucose was apparent. Patients recently withdrawn from neuroactive drugs tended to have lower global metabolic rates, and, this difference, in one instance, attained statistical significance. (Analysis 1: 2- to 8-week drugfree interval, global CMRglu $=7.64 \pm .32 \mathrm{mg}$ glucose $/ 100 \mathrm{~g}$ min; $>1$ year $=8.95 \pm 1.42 ; p=.045$. Analysis 2: 2 - to 8 -week drug-free interval $=7.98 \pm .34$; $>1$ year $=9.17 \pm 1.43 ; p=.07$.) Although metabolic rates did not differ significantly when withdrawal from dopamine receptor antagonists was evaluated independently, these trends persisted.

Therefore, to control for the potentially confounding effects of drug history, regional metabolic rates in TS patients and controls were compared after subjects who had been withdrawn from medications 2 to 8 weeks prior to the study were removed from the analysis. Normalized regional metabolic rates in which significant group differences had previously been detected in both analyses were reevaluated to determine whether differences that had been attributed to diagnosis would persist.

In all but a single instance, differences previously detected between patients and controls were again observed. In analysis 1 , only decreases in metabolism in the left anterior putamen in TS patients failed to attain statistical significance $(p<.09)$. In analysis 2 , all previously detected regional differences persisted when subjects withdrawn from neuroactive medications were excluded. These results suggest that although recent exposure to neuroactive agents may have a global effect on metabolic rates for glucose, such an effect is not responsible for differences attributed to diagnosis in the present analysis.

\section{Estimates of Brain Size and Effects of Gender}

Brain size was estimated by calculating the average cross-sectional area of the image planesselected in analysis 1, excluding the top- and bottom-most slices in each instance to minimize partial volume averaging in the axial plane. There were no significant differences between patients and controls: TS, average cross-sectional area $=100.18 \pm 6.97 \mathrm{~cm}^{2}$, normals $=100.35 \pm 6.59 \mathrm{~cm}^{2}$.

There were no significant differences between average grey matter metabolic rates for glucose calculated for men and women in either analysis (analysis 1: males $=8.63 \pm 1.38 \mathrm{mg}$ glucose $/ 100 \mathrm{~g} / \mathrm{min}$, females $=$ $8.64 \pm .97$; analysis $2:$ males $=8.47 \pm 1.33$, females 
$=8.95 \pm 1.49$ ). Repeated-measures ANOVA performed on values derived from both analyses yielded no significant effects of sex or significant sex by region interactions.

\section{DISCUSSION}

This work attempts to generate, in an exploratory fashion, testable hypotheses regarding the functional neuroanatomy of Tourette's syndrome. Any conclusions drawn from these analyses must be considered preliminary. Our results indicate, nevertheless, that significant differences in regional CMRglu may in fact differentiate TS patients from control subjects. The results of two analyses, each based upon a different technical strategy, suggest that the pathogenesis of this illness may be associated with both increases and decreases in metabolic activity in specific regions of the brain. This pattern suggests, in turn, a neuroanatomic model that could account for the generation of symptoms in this disorder.

In both analyses, decreases in normalized metabolic rates for glucose were detected in ventral limbicassociated regions of the cortex (caudal and lateral orbitofrontal, insular and parahippocampal cortices) and in subcortical regions including the basal ganglia (particularly the ventral striatum, i.e., nucleus accumbens and ventromedial caudate) and the midbrain. These decreases were more robust in the left hemisphere. They appear to be associated with increases in normalized metabolic rates in dorsal sensorimotor regions of the brain, including the supplementary motor area, lateral premotor, and Rolandic cortices. The increases in metabolic activity in these regions were more consistently bilateral. Discriminant analyses suggest that metabolic rates in only this limited set of regions are needed to reliably distinguish TS patients from normal controls.

The reciprocal nature of the differences observed in superior and inferior regions of interest could conceivably represent artifacts of the normalization process itself. Nevertheless, the fact that both sets of changes were detected in either analysis 1 or 2 when absolute metabolic rates were compared suggests that these differences are real.

Differences between TS patients and controls that we observed are not likely to have resulted from common confounding variables such as age, sex, or brain size: estimates of brain size did not differ between patients and controls. The mean, standard deviation, and range of ages in patient and control groups were essentially identical. Although the control group included slightly more females than did the TS patient group, no difference in CMRglu due to sex was detected in either analysis.

Although we cannot entirely rule out the possibil- ity that metabolic changes observed in TS patients could, at least in part, represent the residual effects of chronic dopamine blockade or exposure to other neuroactive medications, the reevaluation of group differences with drug histories taken into account argues against this. Regional differences that were initially detected between patients and controls persisted when subjects who had recently been withdrawn from medications ( 2 to 8 weeks prior to scanning) were excluded from the analysis, leaving only subjects who had been medication-free for a year or longer. This suggests that the effects of recent ( $<1$ year) exposure to neuroactive drugs cannot account for the differences attributed to diagnosis in this study.

Finally, it is not likely that the changes we observed represent the metabolic correlates of motor activity expressed during the scanning procedure. As outlined above, although patients were instructed to remain still during the course of the procedure and were not explicitly instructed to suppress symptoms, vocal or motor tics were only rarely observed during the period of FDG uptake. This seemingly paradoxical decrease in symptoms is not uncommon in TS patients under structured conditions - in a physician's office or classroom and may reflect automatic or unintentional tic suppression. Thus, the present findings could conceivably reflect metabolic changes related to the inhibition of $a b$ normal movements rather than abnormal motor activity per se.

\section{Relationship to Other Functional Neuroimaging Studies}

The present results confirm a preliminary report (Chase et al. 1986) that identified decreases in regional cerebral metabolism in TS patients in caudal, medial, and lateral orbitofrontal cortices, and inferior insula and ventral regions of the basal ganglia. These data were tentatively interpreted as suggesting that striatal and cortical areas associated with the limbic system might be involved in the pathogenesis of TS, a conclusion that is supported and extended by the present findings.

Baxter and Guze (1993) have presented results that appear to differ slightly from our own. These authors report that patients with the concurrent diagnoses of TS and obsessive-compulsive disorder (OCD) showed increased glucose utilization in the putamen when compared to patients with a diagnosis of OCD alone $(n=$ 10; either case) and that all patients with obsessivecompulsive symptoms, whether tics were present or not, had elevated orbitofrontal metabolic rates for glucose when compared to controls. Any differences between these results and our own could be due to the nature of the comparisons, differences in sample size, age, sex, severity of illness, as well as differences in region placement or other methodologic variables. More 
to the point, perhaps, the relationships between cerebral metabolism and obsessive-compulsive symptoms or other complex behavioral features associated with TS have not been addressed in the present study and will be reported in a separate communication (Braun et al. in preparation).

The present findings, however, are by and large consistent with technitium-labeled hexamethyl-propylene amine oxide SPECT studies of regional cerebral blood flow in TS. Riddle et al. (1992) report regional decreases in cerebral perfusion in inferior frontal cortices and lenticular nuclei in nine TS patients (vs. nine normal controls) that appear to be more pronounced in the left hemisphere. Hall et al. (1990) detected hypoperfusion in frontal lobes, mesial temporal structures, and basal ganglia in 25 TS patients using HMPAO. These abnormalities were reported as being frequently asymmetric, although no systematic lateralization is reported. George et al. (1993) evaluated cerebral perfusion with HMPAO in 10 TS patients with and 10 without obsessive-compulsive symptoms, and found increased right frontal/visual cortical activity when the entire cohort of patients was compared to controls $(n=8)$; the frontal region of interest in this study (placed at the level of the basal ganglia) may have included information from both prefrontal and frontal motor cortices.

It is interesting to note that several of the brain regions that appear to be affected in TS patients have been implicated in PET studies of two related illnessesOCD and attention deficit hyperactivity disorder (ADHD). In each instance, however, the direction of these changes (vs. regional control values) is different than that which we observed in TS patients. Obsessivecompulsive disorder appears to be associated with increased metabolic rates in the orbitofrontal cortices and basal ganglia (Baxter et al. 1987; Nordahl et al. 1989; Swedo et al. 1989), and ADHD is associated with decreases in superior sensorimotor structures, centered in the premotor cortices (Zametkin et al. 1990).

It is possible that the same general brain regions are involved in all three of these illnesses, but that the functional interplay between them may differ, accounting for manifest differences in the associated clinical symptoms. Obsessive compulsive disorder and TS, for example, might in some ways be considered antithetical conditions, characterized by motor constraint and discharge, respectively (Cohen et al. 1982). These clinical distinctions could conceivably be related to the respective increases or decreases in orbitofrontal metabolism with which the disorders appear to be associated. In a very simplistic fashion, if the same set of brain regions were to constitute a network involved in a genetic diathesis, differences in the dynamic relationship between these regions might account for the variations in phenotypic expression.

\section{Functional Characteristics of Identified Regions of Interest}

The brain regions in which cerebral metabolic rates differentiate TS patients and controls appear to represent a heterogeneous collection of structures. They are nevertheless functionally related to one another in a way that supports a tentative model of brain dysfunction in TS. The relationship between these regions may normally subserve limbic regulation of motor function.

The superior cortical-premotor and RolandicROIs in which metabolic rates are increased in TS patients are clearly involved in the direct regulation of somatomotor activity, the Rolandic regions mediating supraspinal influences on lower motor neurons in the brainstem and spinal cord (Brodal 1981), and the supplementary motor and lateral premotor cortices involved in associative or integrative functions related to the organization and initiation of complex sequences of movements (Brodal 1981; Fried et al. 1991).

Activity in the premotor cortices is in turn regulated by projections from a variety of cortical and subcortical brain regions. Increased metabolic rates in the frontal motor regions in TS patients in our study are associated with decreases in glucose utilization in an array of ventral regions of the cortex. These inferior cortical areas are in fact constituents of an identified brain system: paralimbic cortices and limbic-related regions of the ventral prefrontal cortex. These regions are in fact derived from a specific subdivision of the paralimbic belt, a paleocortical or "olfactocentric" system centered on the insula (Sanides 1972; Goldberg 1985; Pandya and Barnes 1987).

Paralimbic regions, in general, are interposed between sensorimotor and limbic areas of the brain and may serve as "a synaptic buffer between external reality and internal urges" analogous, in computing terms, to "and/or gates interposed between input and output channels ... allowing the organism to trigger or inhibit behavior" (Mesulam 1985). The paralimbic and inferior prefrontal regions may be involved in monitoring ongoing limbic input to permit impulses to be regulated in accordance with appropriate social norms. (Cummings, 1985).

Paralimbic regions intersect with motor pathways through a variety of routes (Pandya and Yeterian 1985; Mesulam and Mufson 1985; Witter et al. 1989). Nevertheless, the orbital, insular, and mesial temporal cortices are in a position to affect motor function most directly via their monosynaptic projections to the basal ganglia (Heimer and Wilson 1975; DeLong 1983; Alexander et al. 1986), nuclei that play a pivotal role in regulating inhibitory tone in the motor cortices (Chevalier and Deniau 1990). It is to the ventral portion of the striatum that these regions project.

The ventral striatum represents the "limbic-motor interface" (Stevens 1973; Scheel-Kruger and Wilner 
1991). It is the principal site in which information from limbic structures gains access to the motor system (Mogenson 1987; Heimer et al. 1982) in part via interactions with dorsal elements of the core nuclei of the basal ganglia (Haber et al. 1985, 1990). If functional changes in paralimbic regions are linked to changes in the frontal motor cortices in the TS brain, projections through the ventral striatum may be the most direct route through which such coupling might occur. All of these regions may additionally represent elements of one or more of the parallel corticostriatal-thalamocortical circuits proposed by Alexander and coworkers (1986). This issue will be addressed in a separate communication (Braun et al. in preparation).

The involvement of the midbrain is somewhat more difficult to interpret given the functional heterogeneity of this structure and the limited resolution of the PET technique itself. Nevertheless, the midbrain is a critical point of intersection for both limbic and motor systems, and the suggestions that this region may be involved in the pathogenesis of TS has been advanced previously (Devinsky 1983).

\section{Hypotheses}

A model of brain function derived from metabolic data will be useful only if such information is properly interpreted. What, in terms of neuronal activity, do increases and decreases in regional metabolic rates actually represent? Glucose metabolism is clearly related to neuronal firing rates, but the FDG technique does not measure energy consumed in the generation of action potentials in cell bodies of origin. Instead, it measures energy consumed distally, in synaptic elements within the neuropil (Schwartz et al. 1979; Kadekaro et al. 1985). In addition, the FDG technique does not discriminate between excitation and inhibition. At the level of the synapse, both are energy-requiring processes and both produce increases in glucose utilization (Ackerman 1984). The conclusions that can be derived from the analysis of changes in metabolic rates are therefore necessarily limited. The FDG technique simply serves as a reliable marker of synaptic activity, that is, of functional regulation - whether afferent, collateral, or internuncial-of local neuronal activity. Our results can be moreaccurately, if not conclusively, interpreted in this light.

Our data suggest that there is a generalized decrease in synaptic activity within a group of paralimbic and inferior prefrontal structures derived form the paleocortex that may normally serve as a functional buffer between limbic and sensorimotor regions in the brain. These decreases in synaptic regulation are associated with similar reductions in synaptic activity in the ventral striatum: the portion of the basal ganglia to which these regions project, which serves as their mode of entry to the motor system, and which may play a role in regulating inhibitory tone in the frontal motor cortices.

The FDG technique does not indicate whether these changes represent a net attenuation of inhibitory or excitatory tone, and it does not tell us anything about the state of the intrinsic striatal neurons themselves. Nevertheless, a decrease in the flow of information into the ventral striatum and ventromedial caudate appears to be linked, perhaps through interactions within core nuclei of the basal ganglia and thalamus, to an increase in synaptic activity in frontal motor regions of the brain.

These regions, supplementary motor, lateral premotor, and primary motor areas, represent the final common pathway for the initiation of movement and may be associated with what is experienced as intentionality, volitional action, or the "urge" to move (Deecke and Kornhuber 1978; Libet 1985; Goldberg 1987; Fried et al. 1991). The increased synaptic load in these cortical areas could conceivably affect their ability to regulate motor function in a manner that is experienced as voluntary.

Taken together, our results suggest that there may exist a disordered relationship between paralimbic, striatal, and sensorimotor structures, which could in turn reflect a failure of effective limbic-motor integration in the TS brain. The precise mechanisms that might underlie these interactions will be dealt with in a separate communication (Braun et al. in preparation).

Abnormalities in limbic regulation of motor function could account for several characteristic features of TS: the involuntary nature of vocal and motor tics, which are nevertheless associated with the experience of motivational tension; the fact that these symptoms, along with obsessions and compulsions, are often experienced as ego-alien; and the frequent association of motor symptoms with more generalized difficulties in impulse control.

All of these hypotheses need to be rigorously tested. Further studies aimed not at imaging limbic and motor activity in TS patients at rest, but during the execution of normal and abnormal movements, will help to support, extend, or modify the present conclusions.

\section{REFERENCES}

Ackerman RF, Finch DM, BabbTL, Engel J Jr (1984): Increased glucose metabolism during long-duration recurrent inhibition of hippocampal pyramidal cells. J Neurosci 4:251-264

Alexander GE, DeLong MR, Strick PL (1986): Parallel organization of functional segregated circuits linking basal ganglia and cortex. Annu Rev Neurosci 9:357-381

Balthasar K (1956): Uber das anatomishe substrat der generalisierten tic-krankeit (maladie des tics, Gilles de la Tour- 
ette): Entwicklungshemmung des corpus striatum. Arch Psychiatr Nervenkr 195:531-549

Baxter LR, Phelps ME, Mazziotta JC, Guze BH, Schwartz JM, Selin CE (1987): Local cerebral glucose metabolic rates in obsessive compulsive disorder: A comparison with rates in unipolar depression and normal controls. Arch Gen Psychiatry 44:211-218.

Baxter LR, Guze BH (1993): Neuroimaging. In Kurlan R (ed), The Handbook of Tourette Syndrome and OtherTic and Behavioral Disorders. New York, Marcel Dekker, pp 289-304

Bliss J (1980): Sensory experiences of Gilles de la Tourette Syndrome. Arch Gen Psychiatry 37:1343-1347

Brodal A (1981): Neurological Anatomy. New York, Oxford University Press

Braun AR, Stoetter B, Randolph C, Cox C, Hsiao J, Sexton R, Gernert J, Carson RE, Herscovitch P, Chase TN: The functional neuroanatomy of Tourette's syndrome: An FDG PET study; II. Regional cerebral glucose metabolic rates distinguishing clinical subtypes. In Preparation.

Braun AR, Stoetter B, Randolph C, Hsiao J, Pattee L, Gernert J, Carson RE, Herscovitch $P$, Chase TN. The functional neuroanatomy of Tourette's syndrome: An FDG PET study; III. Functional coupling of regional cerebral metabolic rates. In Preparation.

Brooks RA (1982): Alternative formula for glucose utilization using labeled deoxyglucose. J Nucl Med 23:538-539

Brooks RA, Sank VJ, Di Chiro G, Friauf WS, Leighton SB (1980): Design of a high resolution positron emission tomograph: The Neuro-PET. J Comput Assist Tomogr $4: 5-13$

Chase TN, Geoffrey V, Gillespie M, Burrows GH (1986): Structural and functional studies of Gilles de la Tourette Syndrome. Rev Neurol 142:851-855

Chevalier G, Deniau JM (1990): Disinhibition as a basic process in the expression of striatal functions. Trends Neurosci $13: 277-280$

Cohen DJ, Shaywitz BA, Young JG, CarbonariCM, Nathanson JA, Lieberman D, Bowers MB Jr, Maas JW (1979): Central biogenic amine metabolism in children with the syndrome of chronic multiple tics of Gilles de la Tourette: Norepinephrine, serotonin, and dopamine. J Am Acad Child Psychiatry 18:320-341

Cohen DJ, Detlor J, Shaywitz B, Leckman J (1982): Interaction of biological and psychological factors in the natural history of Tourette Syndrome: A paradigm for childhood neuropsychiatric disorders. In Chase TN, Friedhoff AJ (eds), Gilles de la Tourette Syndrome, Advances in Neurology, Vol 35. New York, Raven Press, pp 31-40

Comings DE, Comings BG (1988): Tourette's syndrome and attention deficit disorder. In Cohen DJ, Bruun RD, Leckman JF (eds), Tourette's Syndrome and Tic Disorders: Clinical Understanding and Treatment. New York, Wiley and Sons, pp 119-135.

Cummings JL (1985): Clinical Neuropsychology. Orlando, Grune \& Stratton, pp 57-67

Damasio H, Damasio AR (1989): Lesion Analysis in Neuropsychology. New York, Oxford University Press

Deecke L, Kornhuber HH (1978): An electrical sign of participation of the mesial "supplementary" motor cortex in human voluntary finger movement. Brain Res 159:473-476
DeLong MR, Georgopolis AP, Crutcher MD (1983): Corticobasal ganglia relations and coding of motor performance. In: Massion J, Paillard J, Schultz W, Weisendanger M (eds), Neural Coding of Motor Performance. Experimental Brain Research, Supplement 7. Berlin, SpringerVerlag, pp 30-40

Devinsky O (1983): Neuroanatomy of Gilles de la Tourette's syndrome: Possible midbrain involvement. Arch Neurol 40:508-514

Duvernoy H (1991): The Human Brain Surface, Three-Dimensional Sectional Anatomy and MRI. New York, SpringerVerlag

Fried I, Katz A, McCarthy G, Sass KJ, Williamson P, Spencer SS, Spencer DD (1991): Functional organization of human supplementary motor cortex studied by electrical stimulation. J Neurosci 11:3656-3666

George MS, Trimble MR, Costa DC, Robertson MM, Ring HA, Ell PJ (1993): Elevated frontal cerebral blood flow in Gilles de la Tourette syndrome: A ${ }^{99 m} \mathrm{Tc}-\mathrm{HMPAO}$ SPECT study. Psychiatry Res 45:143-151

Gilles de la Tourette G (1885): Etude sur une affection nerveuse caracreisse par de l'incoordination motrice accompagnee d'echolalie et de copralalie. Arch Neurol 9:19-42, 158-200

Goldberg G (1985): Supplementary motor area structure and function: Review and hypotheses. Behav Brain Sci 8:567616

Goldberg G (1987): From intent to action: Evoluntion and function of the premotor systems of the frontal lobe. In Perecman E (ed), The Frontal Lobes Revisited. New York, IRBN Press, pp 273-306

Haber SN, Groenewegen HJ, Grove EA, Nauta WJH (1985): Efferent connections of the ventral pallidum: Evidence of a dual striato pallidofugal pathway. J Comp Neurol 235:322-335

Haber SN, Kowall NW, Vonsattel JP, Bird ED, Richardson EP (1986): Gilles de la Tourette's syndrome: A postmortem neuropathological and immunohistochemical study. J Neurol Sci 75:225-241

Haber SN, Lynd EL, Mitchell SJA (1990): Comparison between dorsolateral and ventromedial striatal pathways through the monkey basal ganglia. Soc Neurosci Abstr 16:954

Hall M, Costa DC, Shields J, Heavens J, Robertson M, Ell PJ (1990): Brain perfusion patterns with ${ }^{99 \mathrm{~m} T c-H M P A O /}$ SPET in patients with Gilles de la Tourette syndrome. Short report. Nucl Med Suppl 27:243-245

Heimer L, Wilson R (1975): The subcortical projections of the allocortex: Similarities in the neural associations of the hippocampus, the piriform cortex, and the neocortex. In SantiniM (ed), Golgi Centennial Symposium. New York, Raven Press, pp 177-193

Heimer L, Switzer RD, Van Hoesen GW (1982): Ventral striatum and ventral pallidum. Components of the motor system: Trends Neurosci. 5:83-87

Hoffman EJ, Huang SC, Phelps ME (1979): Quantification of positron emission tomography 1: Effect of object size. J Comp Assist Tomogr 3:299-308

Kadekaro M, Crane AM, Sokoloff L (1985): Differential effects of electrical stimulation of sciatic nerve on metabolic activity in spinal cord and dorsal root gangion in the rat. Proc Natl Acad Sci USA 82:6010-6013 
Lang A (1991): Patient perception of tics and other movement disorders. Neurology 41:223-228

Libet B (1985): Unconscious cerebral initiative and the role of conscious will in voluntary action. Behav Brain Sci 8:529-566

Matsui T, Hirano A (1978): An Atlas of the Human Brain for Computerized Tomography. Tokyo, Igaku-Shoin

Meige H, Feindel E (1907): Tics and their Treatment (translated and edited by SAK Wilson). New York, William Wood

Mesulam MM (1985): Patterns in behavioral neuroanatomy: Association areas, the limbic system, and hemispheric specialization. In Principles of Behavioral Neurology. Philadelphia, FA Davis, pp 1-70

Mesulam MM, Mufson E (1985): The insula of Reil in monkey and man: Cytoarchitectonics, connectivity and function. In Peters A, Jones EG (eds), New York, Plenum Press, pp 179-226

Mogenson GJ (1987): Limbic-motor integration. Prog Psychobiol Physiol Psychol 12:117-170

Nordahl TE, Benkelfat C, Semple WE, Gross M, King AC, Cohen RM (1989): Cerebral glucose metabolic rates in obsessive compulsive disorder. Neuropsychopharmacology 2:23-28

Pandya DN, Barnes CL (1987): Architecture and connections of the frontal lobe. In Perecman E (ed), The Frontal Lobes Revisited. New York, IRBN Press, pp 41-72

Pandya DN, Yeterian EH (1985): Architecture and connections of cortical association areas. In Peters A, Jones EG (eds), The Cerebral Cortex. Vol. 4: Association and Auditory Cortices. New York, Plenum Press, pp 3-61

Phelps ME, Huang SC, Hoffman EJ, Selin CE, Sokoloff L, Kuhl DE (1979): Tomographic measurement of local cerebral glucose metabolic rates in humans with (F-18) 2-fluoro-2-deoxyglucose: Validation of method. Ann Neurol 6:371-388

Riddle MA, Hardin MT, Ort SI, Leckman JF, Cohen DJ (1988): Behavioral symptoms in Tourette's syndrome. In Cohen DJ, Bruun RD, Leckman JF (eds), Tourette's Syndrome and Tic Disorders: Clinical Understanding and Treatment. New York, Wiley and Sons, pp 151-162

Riddle MA, Rasmusson AM, Woods SW, Hoffer PB (1992): SPECT imaging of cerebral blood flow in Tourette's syndrome. In Chase TN, Friedhoff AJ, Cohen DJ (eds), Gilles de la Tourette Syndrome: Genetics, Neurobiology and Treatment. Advances in Neurology, Vol. 58. New York, Raven Press, pp 207-211

Rolls ET (1983): The initiation of movements. In Massion J, PaillardJ, Schultz W, Weisendanger M (eds), Neural Coding of Motor Performance Experimental Brain Research, Supplement 7. Berlin, Springer-Verlag, pp 97-113

Rolls ET, Williams GV (1987): Sensory and movement-related neuronal activity in different regions of the striatum of the primate. In Schneider JS, Lidsky TI (eds), Basal Ganglia and Behavior: Sensory Aspects of Motor Functioning. New York, Huber, pp 37-59

Janides F (1972): Representation in the cerebral cortex and its areal lamination patterns. In Bourne $\mathrm{GH}(\mathrm{ed})$, The
Structure and Function of Nervous Tissue, Vol. 5. New York, Academic Press

Scheel-Kruger J, Willner P (1991): The mesolimbic system: Principles of operation. In Willner P, Scheel-Kruger J (eds), The Mesolimbic Dopamine System: From Motivation to Action. New York, Wiley and Sons, pp 559-597

Schwartz WJ, Smith CB, Davidsen L, Savaki H, Sokoloff L, Mata M, Fink DJ, Gainer H (1979): Metabolic mapping of functional activity in the hypothalamoneurohypophysial system of the rat. Science 205:723-725

Seignot MJN (1961): Un cas de maladie des tics de Gilles de la Tourette gueri par le R-1625. Ann Med Psychol 119:578579

Shapiro AK, Shapiro E, Wayne HL (1973): Treatment of Gilles de la Tourette's syndrome with haloperidol: Review of 34 cases. Arch Gen Psychiatry 28:92-96

Singer HS, Hahn IH, Krowiak E, Nelson E, Moran T (1990): Tourette's syndrome: A neurochemical analysis of postmortem brain tissue. Ann Neurol 27:443-446

Singer HS, Tune LE, Butler IJ, Zaczek R, Coyle JT (1982): Clinical symptomatology, cerebrospinal fluid neurotransmitter metabolites, and serum haloperidol levels in Tourette syndrome. In Chase TN, Friedhoff AJ (eds), Gilles de la Tourette Syndrome. Advances in Neurology, Vol 35. New York, Raven Press, pp 185-197

Sokoloff L (1992): Relationship between functional activity and energy metabolism in the nervous system: Whether, where and why. In Ingvar D, Lassen N, Raichle $M$, Friberg L (eds), Brain Work and Energy Metabolism. Proceedings of the Benzon Symposium: Brain Work II. Copenhagen: Munksgaard, pp 52-67

Stevens J (1973): An anatomy of schizophrenia. Arch Gen Psychiatry 177-189

Swedo SE, Schapiro MB, Grady CL, Cheslow DL, Leonard HL, Kumar A, Friedland R, Rapoport SI, Rapoport JL (1989): Cerebral glucose metabolism in childhood-onset obsessive-compulsive disorder. Arch GenPsychiatry 46:518-523

Sweet RD, Solomon GE, Wayne JL, Shapiro E, Shapiro AK (1973): Neurological features of Gilles de la Tourette's syndrome. J Neurol Neurosurg Psychiatry 36:1-9

Towbin KE (1988): Obsessive-compulsive symptoms in Tourette's syndrome. In Cohen DJ, Bruun RD, Leckman JF (eds), Tourette's Syndrome and Tic Disorders: Clinical Understanding and Treatment. New York, Wiley and Sons, pp 137-149

Van de Wetering BJM, Martens CMC, Fortgens C, Slaets JPJ, Van Woerkom RCAM (1985): Late components of the auditory evoked potentials in Gilles de la Tourette's syndrome. Clin Neurol Neurosurg 87:181-186

Witter MP, Groenewegen FH, Lopes Da Silva FH, Lohman AHM (1989): Functional organization of the extrinsic and intrinsic circuitry of the parahippocampal region. Prog Neurobiol 33:161-253

Zametkin AJ, Nordahl TE, Gross M, King AK, Semple WE, Rumsey J, Hamburger S, Cohen RM (1990): Cerebral glucose metabolism in adults with hyperactivity of childhood onset. N Engl J Med 323:1361-1366 